USM-TH-94

\title{
Note on the dual BRST Symmetry in U(1) Gauge Theory
}

\author{
Patricio Gaete* \\ Departamento de Física, Universidad Técnica F. Santa Maria, Valparaíso, Chile
}

\begin{abstract}
We analyze the relation between the Lagrangian and Hamiltonian BRST symmetry generators for a recently proposed two-dimensional symmetry. In particular it is shown that this symmetry may be obtained from a canonical transformation in the ghost sector in a gauge independent way.

PACS number(s): 12.20.-m, 11.30.Ly
\end{abstract}

\section{INTRODUCTION}

Nowadays the concept of BRST symmetry [1] plays an essential role in the quantization of gauge theories. As is well known the BRST formalism has been very useful in the framework of path integral quantization, where the BRST generator (charge) is a key ingredient of the effective action, as well as it finds interesting applications in the operator formulation of the theory. An illustrative example on this subject arises when one considers string theory. In fact, following the BRST inspired approach it was possible to derive the string critical dimensions in a straightforward and economical way [2]. In this context it may be recalled that there exists two approaches to the BRST formulation of gauge theories. One is based on the Hamiltonian formulation, where the BRST charge is constructed in terms of the constraints and the higher order structure functions in a gauge independent way. The

*E-mail: pgaete@fis.utfsm.cl 
other approach is based on the Lagrangian formulation, in such a case the BRST charge is computed from a gauge-fixed Lagrangian by using Noether's prescription. In passing we also recall that in the path integral quantization formalism (both Lagrangian and Hamiltonian) the original gauge invariance is incorporated by means of the extension of the phase space including ghost fields. Thus the main idea is to substitute the local gauge invariance by a rigid Grassmannian symmetry (or global supersymmetry) known as the BRST symmetry. In this way one assigns a global nilpotent charge to this symmetry whose cohomology produces the physical states.

On the other hand, recently a great deal of attention has been devoted to the study of new symmetries in gauge theories. For instance, Lavelle and McMullan [3] found that QED displays a new nonlocal and noncovariant symmetry. In such a case the symmetry transformations are compatible with the gauge-fixing conditions. At the same time Tang and Finkelstein [4] constructed a nonlocal but covariant symmetry for QED. Let us also mention here that Yang and Lee [5] derived a noncovariant but nonlocal symmetry of QED. More recently, Malik [6] showed that in two dimensions of spacetime there exists a local, covariant and nilpotent BRST symmetry, the so-called dual symmetry, under which the gauge-fixing term remains invariant for a free U(1) gauge theory and QED. Furthermore, this author claimed that this symmetry transformation is not the generalization of the above symmetries in two dimensions of spacetime. It is worth stressing at this stage that despite their relevance these studies have been, however, carried out in the gauge fixed scheme only.

Meanwhile, in a previous paper [7] we have discussed the relation between the Lagrangian and Hamiltonian symmetry generators for the Lavelle and McMullan's symmetry in a gauge invariant way using the Batalin-Fradkin-Vilkovisky formalism. In particular, we have showed that the Lavelle and McMullan's symmetry may be derived from a canonical transformation in the ghost sector. We also recall that there are definite advantages of the Hamiltonian approach over the conventional gauge fixed analysis. Ambiguities related to gauge fixing conditions are avoided and it does not need an auxiliary field to construct an off-shell nilpotent symmetry transformation. Let us also mention here that a similar analysis has 
been made, independently, by Rivelles [8]. We are thus motivated to investigate in this paper whether the so-called dual symmetry is a new symmetry or it is merely an artifact of the canonical transformation in the ghost sector.

The outline of this paper is as follows. In Sect.2 we briefly recap the BFV-BRST formalism for a free $\mathrm{U}(1)$ gauge theory in four dimensions of spacetime. This will form the basis of our subsequent considerations. In Sect.3 we will focus our attention to the two-dimensional case. Particular care is paid to establish a direct connection between the Lagrangian and Hamiltonian BRST symmetry generators for the so-called dual symmetry.

\section{GENERAL CONSIDERATIONS ON THE BFV-BRST FORMALISM}

Let us commence our considerations with a short presentation of the BFV-BRST formalism for a free $\mathrm{U}(1)$ gauge theory in four dimensions. It should be noted that this method is a general procedure for quantizing systems with first class constraints. A detailed discussion of the formalism can be found in [2]. We summarize the essence of this formalism in terms of a finite number of phase-space variables, this makes the discussion simpler. In such a case the action for the theory under consideration is taken to be

$$
S=\int d t\left(p^{\mu} \dot{q_{\mu}}-H_{0}-\lambda^{a} \varphi_{a}\right)
$$

where the coordinates $\left(q_{\mu}, p^{\mu}\right)$ are the canonical variables describing the theory. The canonical Hamiltonian is $H_{0}$, and $\lambda^{a}$ are the Lagrange multipliers associated with the first class constraints $\varphi_{a}$. As prescribed by the general theory the Lagrange multipliers are treated in the same foot as the canonical variables, thus we introduce conjugate canonical momenta to $\lambda^{a}$, say $p_{a}$. Evidently, the $p_{a}$ 's must be imposed as new constraints in order that the dynamics of the theory does not change. Now, the BFV approach introduces a pair of canonically conjugate ghosts $\left(C^{a}(x), \mathcal{P}^{a}(x)\right)$ for each constraints. The Poisson algebra of these ghosts is

$$
[C(\mathbf{x}, t), \mathcal{P}(\mathbf{y}, t)]=-\delta(\mathbf{x}-\mathbf{y})
$$


where $C$ and $\mathcal{P}$ has ghost number 1 and -1 , respectively. These considerations naturally lead to an extended phase space, where we have substituted the local gauge invariance by a global supersymmetry invariance (BRST invariance). In this extended phase space the generator of the BRST symmetry for a theory with first class constraints has the form

$$
\Omega=C_{a} \varphi^{a}+\frac{1}{2} P^{a} f_{a}^{b c} C_{b} C_{c}+\ldots
$$

where $f_{a}^{b c}$ are the structure functions, and $\Omega$ is by construction nilpotent $([\Omega, \Omega]=0)$. We also recall that, at the quantum level, in the extended phase space there exists the FradkinVilkovisky theorem [2,9]. This theorem states that the functional integral

$$
\mathcal{Z}_{\Psi}=\int \mathcal{D} \mu \exp \left(i S_{e f f}\right)
$$

where the effective action $S_{\text {eff }}$ is given by

$$
S_{\text {eff }}=\int d t\left(p^{\mu} \dot{q}_{\mu}+C^{a} \dot{\mathcal{P}}_{a}+p^{a} \dot{\lambda}_{a}-H_{0}-[\Omega, \Psi]\right)
$$

being independent of the choice of $\Psi$. Here $\Psi$ is an arbitrary fermionic gauge-fixing function, and $\mathcal{D} \mu$ is the Liouville measure on the phase space. This concludes our brief review of the BFV formalism.

Let us now proceed to apply the above procedure for a free $\mathrm{U}(1)$ gauge theory, in other words,

$$
\mathcal{L}=-\frac{1}{4} F_{\mu \nu} F^{\mu \nu}
$$

Then, from (11) the canonical action takes the form

$$
S=\int d x\left(\dot{A}_{i} \Pi^{i}-H_{0}-\lambda \varphi\right)
$$

where $\Pi^{i}$ is the momenta conjugate to $A_{i} . H_{0}$ is the canonical Hamiltonian, that is,

$$
H_{0}=\int d^{3} x\left(-\frac{1}{2} \Pi_{i} \Pi^{i}+\frac{1}{4} F_{i j} F^{i j}+\Pi_{i} \partial^{i} A_{0}\right)
$$

and it is straightforward to see that the preservation in time of the constraint primary $\left(\Pi^{0}=0\right)$ leads to the secondary constraint 


$$
\varphi=\partial_{i} \Pi^{i}=0 .
$$

We mention in passing that in the action (7) the canonical variables $A_{0}$ and $\Pi^{0}$ have been omitted because $\Pi^{0}=0$, which does not represent a true dynamical degree of freedom of the theory. Thus, $A_{0}$ can be absorbed by redefining the multiplier $\lambda$, i. e., $\lambda$ and $A_{0}$ do not need to be treated as independent variables. With this at hand, the effective action then reads:

$$
S_{e f f}=\int d^{4} x\left(\Pi^{i} \dot{A}_{i}+\mathcal{P} \dot{C}+\overline{\mathcal{P}} \dot{\bar{C}}+\Pi^{0} \dot{A}_{0}-H_{0}-[\Omega, \Psi]\right),
$$

where we have introduced the antighost pair $(\bar{C}(x), \overline{\mathcal{P}}(x))$ with respective ghost numbers -1 and +1 , and satisfying the Poisson algebra (2). The BRST charge $\Omega$ can be easily given as

$$
\Omega=\int d^{3} x\left(C \partial_{i} \Pi^{i}-i \overline{\mathcal{P}} \Pi_{0}\right)
$$

We can now write the corresponding transformations generated by $\Omega$, that is,

$$
\begin{gathered}
\delta A_{i}=-\varepsilon \partial_{i} C, \\
\delta A_{0}=-i \varepsilon \overline{\mathcal{P}} \\
\delta \Pi_{i}=0, \\
\delta \Pi_{0}=0 \\
\delta C=0, \\
\delta \bar{C}=i \varepsilon \Pi_{0}, \\
\delta \overline{\mathcal{P}}=0
\end{gathered}
$$




$$
\delta \mathcal{P}=-\varepsilon \partial_{i} \Pi^{i}
$$

where $\varepsilon$ is an anticommuting spacetime independent infinitesimal parameter. In order to compute the effective action (10), we have to select the gauge fixing function $\Psi$. There are a variety of these which have been found useful and convenient in different calculational context. We can choose, for example, $\Psi$ in the form

$$
\Psi=\int d^{3} x\left(\mathcal{P} A_{0}-i \bar{C}\left(\frac{x_{i} A^{i}}{x^{2}}-\frac{\xi}{2} \Pi^{0}-\dot{A_{0}}\right)\right),
$$

which leads to the modified Fock-Schwinger gauge [10]. However, of this turn, we take $\Psi$ as

$$
\Psi=\int d^{3} x\left(\mathcal{P} A_{0}-i \bar{C}\left(\partial_{i} \Pi^{i}-\frac{\xi}{2} \Pi_{0}\right)\right),
$$

where $\xi$ is a real parameter that describes a set of gauges. Explicitly, for $\xi=0,1$ and infinity we obtain the Landau, Feynman and unitary gauges, respectively. Plugging this expression into (10), we find that the resulting effective action is given by

$$
S_{e f f}=\int d^{4} x\left(-\frac{1}{4} F^{\mu \nu} F_{\mu \nu}+i \bar{C} \partial_{\mu} \partial^{\mu} C+\frac{1}{2 \xi}\left(\partial_{\mu} A^{\mu}\right)^{2}\right) .
$$

We immediately recognize the above to be the same as the Lagrangian effective action.

Before concluding this section we call attention to the fact that in contrast to the gaugefixing term, the gauge field $F_{\mu \nu}$ remains invariant under the transformation generated by $\Omega$ (11), that is, $\delta F_{\mu \nu}=0$ and $\delta\left(\partial_{\mu} A^{\mu}\right)=-\varepsilon\left(i \dot{\overline{\mathcal{P}}}-\nabla^{2} C\right)$. However, it is possible to recast the BRST charge (11) which corresponds to a nilpotent symmetry transformation under which the gauge-fixing term remains invariant. This can be done by a suitable canonical transformation in the BFV phase space, in such a way that any two BRST generators are related by such transformations [2]. In the work of Ref. [7], we had showed that by performing the following canonical transformation in the ghost sector:

$$
\begin{aligned}
& C^{\prime}=\frac{1}{\nabla^{2}} \mathcal{P}, \\
& \mathcal{P}^{\prime}=\nabla^{2} C,
\end{aligned}
$$




$$
\begin{aligned}
& \bar{C}^{\prime}=-\overline{\mathcal{P}}, \\
& \overline{\mathcal{P}}^{\prime}=-\bar{C},
\end{aligned}
$$

the gauge-fixing term remains invariant. In effect, as a consequence of this canonical transformation, the new BRST charge $\Omega^{\perp}$ then becomes

$$
\Omega^{\perp}=\int d^{3} x\left(\frac{1}{\nabla^{2}} \mathcal{P} \partial_{i} \Pi^{i}+i \bar{C} \Pi_{0}\right) .
$$

Hence we see that the corresponding transformations generated by $\Omega^{\perp}$ are:

$$
\begin{gathered}
\delta^{\perp} A_{i}=-\varepsilon \partial_{i} \frac{1}{\nabla^{2}} \mathcal{P}, \\
\delta^{\perp} A_{0}=i \varepsilon \bar{C}, \\
\delta^{\perp} \Pi_{\mu}=0, \\
\delta^{\perp} C=-\varepsilon \frac{1}{\nabla^{2}} \partial_{i} \Pi^{i}, \\
\delta^{\perp} \bar{C}=0, \\
\delta^{\perp} \mathcal{P}=0, \\
\delta^{\perp} \overline{\mathcal{P}}=-i \varepsilon \Pi_{0} .
\end{gathered}
$$

Thus it follows that on integration over the momenta the gauge-fixing term remains invariant under the transformation generated by $\Omega^{\perp}$, that is, $\delta^{\perp}\left(\partial_{\mu} A^{\mu}\right)=0$. The above expressions coincide with the Lavelle and McMullan's result [3]. However, these symmetry transformations turn out to be nonlocal. The preceding analysis opens up the way to a stimulating discussion of how the so-called dual BRST symmetry appears. This is precisely the task that we shall carry out in the next section. 


\section{DUAL BRST SYMMETRY}

As already mentioned, our immediate objective is to implement the above general considerations to the two-dimensional case. With this in mind, we start by considering

$$
\mathcal{L}=-\frac{1}{4} F_{\mu \nu} F^{\mu \nu}
$$

in two dimensions of spacetime. Just as for the four-dimensional case, the canonical action is

$$
S=\int d x\left(\dot{A_{1}} \Pi^{1}-H_{0}-\lambda \varphi\right)
$$

where $\Pi^{1}$ is the momenta conjugate to $A_{1}$. Here it is important to realize that the corresponding canonical Hamiltonian is now

$$
H_{0}=\int d x\left(-\frac{1}{2} \Pi_{1} \Pi^{1}+\Pi_{1} \partial^{1} A_{0}\right)
$$

The constraint structure for the gauge field naturally remains identical to the previous case ( See Eq. (9) ). Thus it follows that

$$
\varphi=\partial_{1} \Pi^{1}=0
$$

Again we find that the effective action can be written as

$$
S_{e f f}=\int d x\left(\Pi^{1} \dot{A}_{1}+\mathcal{P} \dot{C}+\overline{\mathcal{P}} \dot{C}+\Pi^{0} \dot{A}_{0}-H_{0}-[\Omega, \Psi]\right)
$$

As in the preceding section, the BRST generator reduces to

$$
\Omega=\int d x\left(C \partial_{1} \Pi^{1}-i \overline{\mathcal{P}} \Pi_{0}\right)
$$

We can now write the corresponding transformations generated by $\Omega$, that is,

$$
\begin{gathered}
\delta A_{1}=-\varepsilon \partial_{1} C, \\
\delta A_{0}=-i \varepsilon \overline{\mathcal{P}}
\end{gathered}
$$




$$
\begin{gathered}
\delta \Pi_{1}=0, \\
\delta \Pi_{0}=0, \\
\delta C=0, \\
\delta \bar{C}=i \varepsilon \Pi_{0}, \\
\delta \overline{\mathcal{P}}=0, \\
\delta \mathcal{P}=-\varepsilon \partial_{1} \Pi^{1} .
\end{gathered}
$$

Following our procedure we now calculate the effective action (39). As in the previous section, we choose the gauge-fixing function in the form

$$
\Psi=\int d x\left(\mathcal{P} A_{0}-i \bar{C}\left(\partial_{1} A^{1}-\frac{\xi}{2} \Pi_{0}\right)\right) .
$$

In the present case, the effective action is found to be

$$
S_{e f f}=\int d^{2} x\left(-\frac{1}{4} F_{\mu \nu} F^{\mu \nu}-\frac{1}{2 \xi}\left(\partial_{\mu} A^{\mu}\right)^{2}+i \bar{C} \partial_{\mu} \partial^{\mu} C\right) .
$$

It has been recently claimed [6] that the effective action is also invariant under local variations:

$$
\begin{gathered}
\delta_{D} A_{\mu}=-\eta \varepsilon_{\mu \nu} \partial^{\nu} \bar{C}, \\
\delta_{D} \bar{C}=0, \\
\delta_{D} C=-i \eta \mathcal{B},
\end{gathered}
$$

where $\mathcal{B}$ is an auxiliary field. Accordingly, we have that

$$
\delta_{D}\left(\partial_{\mu} A^{\mu}\right)=0 .
$$


At this point it is reasonable to ask how the transformations (51- 53) are related with the ones (41-48). In view of this situation and on the basis of the discussion in the previous section, we now proceed to perform a canonical transformation in the ghost sector. In that case, we propose the following canonical transformation

$$
\begin{gathered}
C^{\prime}=i \frac{\mathcal{P}}{\partial_{1}}, \\
P^{\prime}=-i \partial_{1} C, \\
\bar{C}^{\prime}=-i \frac{\overline{\mathcal{P}}}{\partial^{1}} \\
\bar{P}^{\iota}=i \partial^{1} \bar{C} .
\end{gathered}
$$

As before, we keep the notation $\Omega^{\perp}$ for the charge which results from a canonical transformation. Thus, the new charge may be rewritten as

$$
\Omega^{\perp}=\int d x\left(i \frac{\mathcal{P}}{\partial_{1}}\left(\partial_{1} \Pi^{1}\right)-\partial^{1} \bar{C} \Pi_{0}\right)
$$

It is now once again straightforward to work out the transformations generated by (59). They are

$$
\begin{gathered}
\delta^{\perp} A_{1}=i \varepsilon \mathcal{P}, \\
\delta^{\perp} A_{0}=-\varepsilon \partial^{1} \bar{C}, \\
\delta^{\perp} \Pi_{\mu}=0, \\
\delta^{\perp} \bar{C}=0, \\
\delta^{\perp} C=-i \varepsilon \Pi^{1}, \\
\delta^{\perp} \mathcal{P}=0,
\end{gathered}
$$




$$
\delta^{\perp} \overline{\mathcal{P}}=-\varepsilon \partial^{1} \Pi_{0}
$$

One immediately sees that, on integration over the momenta, the above transformations (6066]) reduce to the ones found in [6]. It is important to realize that, after integration over the momenta, the new transformations yield $\delta^{\perp}\left(\partial_{\mu} A^{\mu}\right)=0$ off shell. It is worthwhile sketching at this point our procedure. As mentioned before, in the extended phase space we have

$\delta\left(\partial_{\mu} A^{\mu}\right)=-\varepsilon\left(i \dot{\mathcal{P}}+\partial_{1} \partial^{1} C\right)$, which in the configuration space reads $\delta\left(\partial_{\mu} A^{\mu}\right)=\varepsilon \partial_{\mu} \partial^{\mu} C$, but this is just the classical equation of motion of $C$. From our above analysis, we see that the proposed canonical transformation makes a change of the ghost equations, that is, $\delta^{\perp}\left(\partial_{\mu} A^{\mu}\right)=-\varepsilon \partial^{1}(\overline{\bar{C}}-i \mathcal{P})$ which after integration over the momenta gives zero, turning the variation of the gauge-fixing term null on shell to null off shell. Since the canonical transformation has been carried out in the ghost sector, all the basics processes that can be explained by the old effective action, should likewise be obtained from the new effective action. It is satisfying to notice the simplicity and directness of this derivation, which is manifestly gauge-independent.

\section{ACKNOWLEDGMENTS}

The author would like to thank I. Schmidt for his support, and J.J. Yang for reading the manuscript. Work supported in part by Fondecyt (Chile) grant 1000710, and by a Cátedra Presidencial (Chile). 


\section{REFERENCES}

[1] C. Becchi, A. Rouet, and R. Stora, Phys. Lett. 52B, 344 (1974); Ann. Phys. (N.Y.) 98, 287 (1976); I. V. Tyutin, Lebedev Report N FIAN 39, 1975 (unpublished).

[2] M. Henneaux and C. Teitelboim, Quantization of Gauge Systems (Princeton University Press, Princeton, 1992); M. Henneaux, Phys. Rep. 126, 1 (1985).

[3] M. Lavelle and D. McMullan, Phys. Rev. Lett. 71, 3758 (1993).

[4] Z. Tang and D. Finkelstein, Phys. Rev. Lett. 73, 3055 (1994).

[5] H. Yang and B.-H. Lee, J. Math. Phys. 37, 6106 (1996).

[6] R. P. Malik, hep-th/9711056, hep-th/9902146 and hep-th/0003128.

[7] P. Gaete and S. J. Rabello, Phys. Rev. D52, 7205 (1995).

[8] V. O. Rivelles, Phys. Rev. D53, 3247 (1996).

[9] E. S. Fradkin and G. Vilkovisky, Phys. Lett. B55, 224 (1975); I. A. Batalin and G. Vilkovisky, Phys. Lett. B69, 309 (1977).

[10] J. Barcelos-Neto, P. Gaete and C. A. P. Galvão, Mod. Phys. Lett. A17, 1597 (1991). 